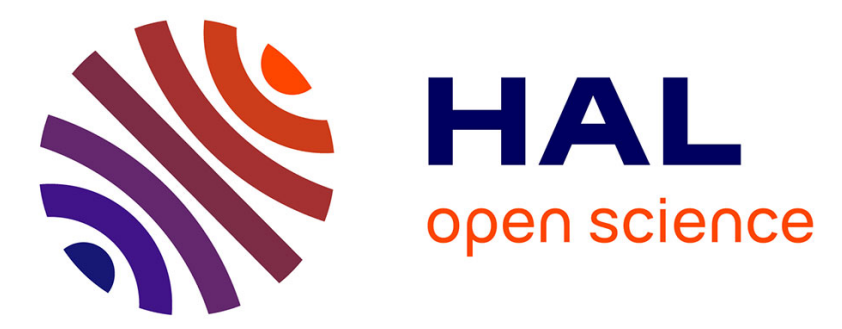

\title{
L'ethnographie comme démarche compréhensive : immersion dans les dynamiques consommatoires du rap en France
}

Baptiste Cléret

\section{- To cite this version:}

Baptiste Cléret. L'ethnographie comme démarche compréhensive: immersion dans les dynamiques consommatoires du rap en France. Recherches Qualitatives, 2013. halshs-01597621

\section{HAL Id: halshs-01597621 https://shs.hal.science/halshs-01597621}

Submitted on 28 Sep 2017

HAL is a multi-disciplinary open access archive for the deposit and dissemination of scientific research documents, whether they are published or not. The documents may come from teaching and research institutions in France or abroad, or from public or private research centers.
L'archive ouverte pluridisciplinaire $\mathbf{H A L}$, est destinée au dépôt et à la diffusion de documents scientifiques de niveau recherche, publiés ou non, émanant des établissements d'enseignement et de recherche français ou étrangers, des laboratoires publics ou privés. 


\title{
L'ethnographie comme démarche compréhensive : immersion dans les dynamiques consommatoires du rap en France
}

Baptiste Cléret, Maître de conférences

\author{
Université de Rouen
}

\begin{abstract}
Résumé
Initialement fondée sur des perspectives quantitativistes, la recherche en sciences de gestion compte un nombre croissant de démarches qualitatives, dont le fondement réside davantage dans la compréhension d'un phénomène que dans son explication. Parmi l'éventail de protocoles qualitatifs à disposition des chercheurs, l'ethnographie représente un outil pertinent tant dans une perspective de collecte que d'analyse des données. L'objectif de cet article est de proposer une présentation de la démarche ethnographique et de sa pertinence en sciences de gestion, plus particulièrement en marketing. À partir d'une recherche sur le mouvement rap français, cet article s'articule autour, d'une part, d'une présentation de l'ethnographie, de ses fondements et de ses principes, et, d'autre part, de son application dans le cadre d'une recherche en comportement du consommateur.

Mots clés

ETHNOGRAPHIE, THÉORIES CULTURELLES DE LA CONSOMMATION, ÉTUDES

QUALITATIVES, MARKETING
\end{abstract}

\section{Introduction}

La complexité de nos sociétés contemporaines et des comportements de consommation en particulier, à l'heure de la mondialisation et de la numérisation des pratiques, amène les chercheurs en sciences de gestion à s'interroger sur les méthodes à employer afin d'appréhender des phénomènes. Parmi l'ensemble d'outils à disposition des chercheurs, l'ethnographie apparaît de plus en plus comme une démarche d'investigation pertinente pour saisir de façon fine les pratiques de consommation et le contexte socioculturel dans lequel celles-ci naissent, se diffusent et se transforment.

Définie le plus souvent comme une méthode d'investigation permettant de réaliser la «description dense » (Geertz, 1973, p. 27) d'un fait social, l'ethnographie est apparue au sein des sciences humaines comme une démarche

RECHERCHES QUALITATIVES - Vol. 32(2), pp. 50-77.

LA RECHERCHE QUALITATIVE DANS LES SCIENCES DE LA GESTION. DE LA TRADITION À L'ORIGINALITÉ

ISSN 1715-8702 - http://www.recherche-qualitative.qc.ca/Revue.html

(C) 2013 Association pour la recherche qualitative 
pertinente et capable d'apporter des éléments de compréhension des sociétés, des cultures et des activités humaines. Initialement utilisée pour décrire des cultures éloignées, l'ethnographie est devenue, au milieu du $\mathrm{XX}^{\mathrm{e}}$ siècle, une méthode d'investigation pertinente pour étudier des populations ou des communautés urbanisées. Les sciences de gestion, et particulièrement le marketing, ont intégré, par la suite, cette approche dans l'éventail méthodologique disponible à toute démarche qualitative afin d'appréhender des comportements de consommation en constante évolution.

Ainsi, cet article présente, dans un premier temps, les fondements de l'ethnographie, de la compréhension de sociétés dites primitives à l'appréhension de dynamiques consommatoires. Nous exposons les principes fondamentaux de la démarche ethnographique ainsi que la place et le rôle du chercheur dans cette méthode d'investigation. Puis, dans un second temps, afin de proposer une illustration concrète de cet outil, nous analysons la mise en place de l'ethnographie dans le cadre d'une recherche en comportement du consommateur centrée sur une compréhension contextualisée des comportements de consommation culturelle des jeunes au sein du mouvement rap français.

Des sociétés lointaines aux cultures de consommation : itinéraire et fondements de l'ethnographie

Apparu à la fin du XVIII ${ }^{\mathrm{e}}$ siècle, le terme ethnographie, du grec ethnos (« groupe, peuple») symbolise, à l'origine, le classement des langues (Copans, 1996). C'est au cours du XIX ${ }^{\mathrm{e}}$ siècle que l'ethnographie prend son sens commun, c'est-à-dire « la description des faits » (Copans, 1996, p. 9).

La particularité de l'ethnographie réside dans son caractère visuel. À ce titre, Laplantine (2006) précise que «l'ethnographie est d'abord une activité visuelle ou, comme le disait Marcel Duchamp de la peinture, une activité "rétinienne" » (p. 9). Cette particularité visuelle de la méthode ethnographique vient notamment des travaux de Malinowski (1963, 1985), imposant l'observation directe de la « vie réelle » (Malinowski, 1963, p. 74) comme un des principes fondateurs de toute recherche ethnographique. Dans les années 1970, Clifford Geertz conçoit l'ethnographie comme une technique permettant de lire la culture comme un texte (Geertz, 1998), une méthode d'enquête basée sur la compréhension d'un corps étranger au chercheur (Mauss, 1926) que ce dernier tente de déchiffrer afin d'en faire ressortir l'essence.

Itinéraire de la démarche ethnographique

Ainsi, l'ethnographie représente une méthode permettant de rendre compte de faits sociaux en mettant en place une « étude complète des phénomènes, et non 
pas à une recherche du sensationnel, de l'original, encore moins de l'amusant et du bizarre » (Malinowski, 1963, p. 68). C'est en ce sens que l'ethnographie a été investie pour comprendre des cultures éloignées, puis des phénomènes de consommation.

Ethnographie, ethnologie et anthropologie : étude de l'homme dans son milieu naturel

Afin de comprendre l'essence même de l'ethnographie, il convient de distinguer cette dernière de deux termes voisins et interreliés : ethnologie et anthropologie.

L'ethnologie symbolise «la science qui reconstitue l'histoire des peuples » (Copans, 1996, p. 8), une analyse ou une interprétation de la description ethnographique. Malinowski (1963) la distingue également de l'ethnographie, employant «le terme ethnographie pour les résultats empiriques et descriptifs de la science de l'Homme, et le mot ethnologie pour les théories spéculatives et comparatives » (p. 65). Dès lors, d'après ces deux auteurs, l'ethnographie représente une méthode permettant de rendre compte de faits sociaux alors que l'ethnologie incarne davantage l'analyse de celle-ci. Au centre de ces deux concepts, l'anthropologie représente différentes disciplines en tant qu' "étude de l'homme tout entier» (Laplantine, 2006, p. 9) ou de «science sociale et culturelle générale de l'homme»(Copans, 1996, p. 8). L'anthropologie s'intéresse donc aux activités de l'homme "dans toutes les sociétés, sous toutes les latitudes, dans tous ses états et à toutes les époques » (Laplantine, 2006, p.9). En d'autres termes, l'ethnographie représente une méthode d'investigation dont la finalité est de proposer un «tableau descriptif » de la dimension culturelle d'un groupe social, dont l'analyse mettra à jour les fondements.

C'est dans cette première appréhension de l'ethnographie que des auteurs tels que Mauss (1926), Malinowski (1963) ou Geertz (1973) ont tenté de saisir l'essence de cultures et de sociétés éloignées. L'École de Chicago, puis le courant des Cultural Studies, prolongeront la réflexion ethnographique en employant cet outil dans des contextes urbains.

Naissance d'une ethnographie urbaine : de Chicago à Birmingham

Si l'ethnologie a d'abord été appliquée à la compréhension des sociétés dites primitives, elle a ensuite été pratiquée dans des cadres contemporains et urbains dont l'École de Chicago fut pionnière'. Afin d'enrichir sa description d'éléments signifiants, l'École de Chicago puise notamment son inspiration dans le courant de l'interactionnisme symbolique, développé par Georges Herbert Mead à la fin du XIX ${ }^{\mathrm{e}}$ siècle, dont l'objectif est de souligner « la nature symbolique de la vie sociale»(Coulon, 1992, p. 14). En développant une 
approche centrée sur l'étude de communautés, les chercheurs de l'École de Chicago ont construit des cartographies ethnographiques des territoires culturels et sociaux des villes au sein de leurs diverses populations (Blackman, 2005), en développant les prémisses d'une théorie sous-culturelle grandement enrichie par le courant des Cultural Studies.

En Grande Bretagne, les chercheurs de Birmingham, berceau du courant des Cultural Studies, s'inscrivent dans les travaux de l'École de Chicago en entreprenant, notamment, des investigations ethnographiques de groupements juvéniles (Encadré 1).

Ethnographie et marketing : compréhension contextualisée des comportements de consommation

Lors de son expansion et de son développement, le marketing s'est régulièrement tourné vers les méthodes et les théories des sciences sociales afin de comprendre et d'expliquer davantage les comportements de consommation des individus. Afin de rendre compte de ces pratiques de plus en plus diffuses et complexes (Dion, 2008), les chercheurs en marketing ont trouvé dans les méthodes qualitatives des outils adaptés. Parmi celles-ci, la méthode ethnographique apparaît comme une perspective méthodologique pertinente permettant de comprendre de façon fine les dynamiques consommatoires actuelles.

L'adaptation de la méthode ethnographique à des problématiques marketing s'est faite de multiples façons à partir de phénomènes tels que l'étude de communautés de consommation (Kates, 2002; Schouten \& McAlexander, 1995), des dimensions ethniques de la consommation (Peñaloza, 1994), des comportements en points de vente (Badot, 2005; Peñaloza, 1999) ou des dynamiques consommatoires juvéniles (Ezan, 2009; Kjelgaard \& Askegaard, 2006; Ritson \& Elliot, 1999).

Ces diverses applications illustrent le recours croissant aux méthodes qualitatives, particulièrement aux approches ethnographiques, dont la Consumer Behavior Odyssey de Belk, Wallendorf et Sherry (Belk, 1987; Belk et al., 1989) incarne le symbole d'un tournant interprétatif dans les dispositions méthodologiques de recherche en comportement de consommation. Depuis une dizaine d'années, ces travaux portant un regard ethnographique et socioculturel sur la consommation contemporaine et partageant la même volonté de mettre à jour les « aspects socioculturels, expérientiels, symboliques et idéologiques de la consommation » (Arnould \& Thompson, 2005, p. 868) se regroupent au sein $\mathrm{du}$ courant de recherche de la Consumer Culture Theory (Arnould \& Thompson, 2005). 
Au carrefour de disciplines diverses telles que la philosophie, la sociologie ou l'ethnologie, le courant des Cultural Studies a adapté les méthodes ethnographiques développées par l'École de Chicago pour tenter de comprendre la formation de groupements juvéniles appelés « sous-cultures ». Des ouvrages tels que Resistance through rituals (Hall \& Jefferson, 1976) ou Subculture : the meaning of style (Hebdige, 1979) ont contribué à asseoir la démarche ethnographique anglo-saxonne. D'un point de vue méthodologique, Phil Cohen (1972) argue que la compréhension de ces sous-cultures à partir d'une approche ethnographique passe nécessairement par trois niveaux d'analyse :

- Une analyse historique qui isole la problématique spécifique d'un groupe social particulier, «d'une fraction de classe », distinct des autres;

- Une analyse sémiotique ou structurelle de sous-systèmes représentant la manière dont ils sont articulés et les transformations réelles que ces sous-systèmes subissent d'un moment structurel à un autre;

- Une analyse phénoménologique de la façon dont la sous-culture est vécue en dehors par ceux qui sont les « porteurs » et les supports de ces sous-cultures.

Encadré 1. Cultural studies et ethnographie : étude des sous-cultures juvéniles.

Appliquée au marketing, l'ethnographie peut être considérée comme « une perspective théorique qui met l'accent sur le concept de culture et sa relation avec le comportement observé, et le principal outil analytique pour classer et expliquer les dynamiques consommatoires » (Mariampolsky, 2006, p. 6). Cet auteur ajoute à cette première appréhension de l'ethnographie une deuxième définition dans laquelle l'ethnographie est à la fois l'outil méthodologique et l'analyse des données récoltées. Cette compréhension relativement plurielle de l'outil ethnographique a donné lieu à de multiples adaptations de cet outil en marketing (Encadré 2).

Qu'elle soit vue comme une méthode descriptive ou comme le résultat de celle-ci, l'ethnographie se caractérise par des principes fondateurs lui permettant à la fois de se distinguer d'autres outils de recherche, mais également d'asseoir une certaine rigueur scientifique. 
Afin de transposer l'outil ethnographique à des problématiques marketing, certains auteurs ont proposé des perspectives méthodologiques spécifiques sur la base de la logique ethnographique. En France, l'application de l'approche ethnographique aux recherches en marketing s'est faite à partir des travaux de Badot, Carrier, Cova, Desjeux et Filser (2009), en proposant le concept d'ethnomarketing comme démarche anthropologique de la consommation et en se basant sur une posture «d'induction cadrée» (Badot et al., 2009, p. 100).

Par ailleurs, afin d'adapter la démarche ethnographique à la prédominance du média Internet dans la sphère consommatoire contemporaine, Kozinets (1997, 2002) a développé l'outil netnographique, conçu comme une méthode ethnographique appliquée à Internet ${ }^{2}$.

Enfin, des chercheurs en marketing ont également proposé une adaptation de l'outil ethnographique à l'émergence de relations et de conditions de marchés globalisés (Abélès, 2008; Appaduraï, 1990), à partir de l'ethnographie multisites (Hannerz, 2003; Marcus, 1995). Dans cette perspective, l'ethnographie a pour objectif «à travers l'étude de multiples sites locaux, d'étendre la portée de l'ethnographie en incluant non seulement la vie des individus mais aussi les associations et les connexions entre les sites qui composent le système » (Kjelgaard, Faurholt Csaba, \& Ger, 2006, p. 525).

Encadré 2. Adaptations de l'ethnographie aux évolutions consommatoires.

\section{Principes ethnographiques : le chercheur comme partie prenante}

La démarche ethnographique a pour particularité de se baser sur une participation importante du chercheur dans le phénomène étudié. Que son immersion soit partielle ou complète, le chercheur-ethnographe ne peut être détaché de son objet d'étude. La subjectivité est alors partie intégrante de toute ethnographie. Après avoir présenté les principes fondateurs de l'ethnographie en général et leur application au marketing en particulier, la partie suivante abordera la place du chercheur dans la démarche ethnographique ainsi que les dispositions à prendre pour que son immersion personnelle enrichisse son investigation.

Fondements méthodologiques de l'ethnographie

Comme il a été précisé précédemment, l'ethnographie a pour but de lire une culture comme un texte (Geertz, 1973) afin de comprendre un corps étranger au chercheur (Mauss, 1926). Cette compréhension passe indubitablement par le respect de certains principes dont l'immersion personnelle de l'ethnographe incarne le cœur. 
Poursuivant dans ce sens, Malinowski (1963) précise que l'ethnographe doit prendre en considération trois approches s'il veut atteindre ses objectifs :

- «L'organisation de la tribu ainsi que l'anatomie de sa culture doivent être fixées sous forme de canevas clairs et précis » (Malinowski, 1963, p. 81). Une des premières missions de toute approche ethnographique est donc de décrire, le plus précisément possible, la structuration du phénomène étudié;

- «[L]es impondérables de la vie authentique et le type de comportement doivent être insérés. Il convient de recueillir ces éléments par des observations minutieuses, détaillées, sous forme d'une espèce de journal ethnographique, rendues possibles par un contact étroit avec l'existence indigène $»^{3}$ (Malinowski, 1963, p. 81);

- «[U]ne collection de rapports ethnographiques, récits caractéristiques, expressions typiques, faits folkloriques et formules magiques, doit former un corpus inscriptionum servant de témoignage sur la mentalité indigène » (Malinowski, 1963, p. 81). La constitution d'un corpus de données multiples et variées est donc nécessaire à la description du phénomène étudié.

Si Mauss (1926) et Malinowski (1963) ont été les premiers à proposer un protocole précis de la démarche ethnographique dans lequel l'observation et la participation du chercheur représentent les piliers, Van Maanen (1979) a milité en faveur d'une ethnographie organisationnelle. Pour ce faire, Van Maanen (1979), illustrant son propos à l'aide d'une étude sur la police, précise que tout chercheur pratiquant l'ethnographie doit être en mesure de distinguer les concepts de premier ordre (représentant les faits) et les concepts de second ordre (désignant l'interprétation du chercheur et les théories mobilisées afin d'organiser et d'expliquer les faits). On retrouve ici la distinction entre le récit descriptif des faits observés et l'analyse mise en place par le chercheur pour saisir le sens de ces faits.

Appliquée au marketing et à la recherche sur le comportement du consommateur, Arnould et Wallendorf (1994) proposent le terme d'ethnographie orientée marché défini comme « une approche ethnographique centrée sur les comportements des individus constituant un marché pour un bien ou un service » (p. 484). L'ethnographie permet alors de se démarquer de la perspective cognitiviste en comprenant les actions individuelles à travers les motifs sociaux et culturels et en prenant en compte les contextes sociaux dans lesquels ces actions se réalisent (Ritson \& Elliot, 1999). Afin d'appliquer la démarche ethnographique dans un contexte de marché, Arnould et Wallendorf (1994) distinguent quatre caractéristiques qui différencient une recherche 
ethnographique d'une autre, mais qui assoient également la légitimité et la scientificité de cette démarche :

- La collecte de données dans leur cadre naturel;

- La participation expérientielle longue de la part du chercheur, notamment à partir d'observations participantes;

- Les interprétations issues du travail ethnographique doivent être crédibles aux yeux des personnes étudiées et de l'audience;

- L'ethnographie suppose un recours à des sources de données qui sont multiples et un intérêt porté à d'autres domaines de recherche en sciences sociales afin de générer des perspectives variées sur les comportements et le contexte.

Ainsi, l'ethnographie se différencie des autres outils qualitatifs par une mise en exergue de la nécessaire participation, voire l'immersion, du chercheur dans le phénomène qu'il cherche à comprendre. Cette spécificité ethnographique pose la question, d'une part, de l'entrée du chercheur dans l'univers socioculturel qu'il tente de saisir, mais également, d'autre part, des biais et de la valeur ajoutée que cette participation personnelle engendre.

La place du chercheur dans la démarche ethnographique: entre richesse scientifique et contamination

Le chercheur est donc partie intégrante de toute recherche ethnographique. Comme le soulignent Arnould et Wallendorf (1994, p. 485), «la recherche ethnographique implique la participation expérientielle étendue de la part du chercheur dans un contexte culturel spécifique ». Le chercheur a pour objectif de comprendre ce qu'il étudie en s'y investissant personnellement. Lorsque le chercheur a identifié le groupe social, la (sous-)culture ou le territoire qu'il souhaite étudier, il se prépare à s'immerger, de façon plus ou moins profonde, dans le quotidien des individus qui y vivent, tel un plongeur s'apprêtant à découvrir des fonds marins (Encadré 3).

«Le jeune ethnographe qui part sur le terrain doit savoir ce qu'il sait déjà, afin d'amener à la surface ce qu'on ne sait pas encore », nous dit Mauss (1926, p. 6). Il doit ainsi être en éveil constant afin d'affiner sa perception de l'objet d'étude et de faire émerger des éléments de compréhension en lien avec sa ou ses questions de recherche.

L'entrée sur le terrain est une phase délicate pour tout chercheur souhaitant entreprendre une démarche ethnographique. Son statut de chercheur 
L'immersion de l'ethnographe dans un phénomène qui lui est inconnu peut être comparée à un plongeur se préparant à découvrir de nouveaux trésors dans le fond des océans. À l'image du plongeur qui vérifie les coordonnées de sa zone de plongée sur une carte, l'ethnographe délimite son champ d'études. Quand le plongeur contrôle ses bouteilles, vérifie son masque et son altimètre, l'ethnographe examine son matériel de recherche (carnet de note, appareil photo, dictaphone), planifie et choisit ses outils de collecte de données. La phase de préparation terminée, le plongeur et l'ethnographe peuvent prendre part à l'exercice qui leur incombe, soit s'immerger dans un environnement presque inconnu afin de saisir ce qui en est le cœur et l'essence.

Encadré 3. L'immersion ethnographique du chercheur: la métaphore du plongeur.

ou «d'étranger» au groupe ou au phénomène étudié peut se traduire en une forme de réticence, de la part des individus, à voir arriver une personne extérieure. Pour légitimer cette entrée, certaines recherches, à l'image de celle de Whyte (1955) sur la structure sociale d'un quartier italo-américain, ont recours à un interlocuteur privilégié qui joue le rôle de «parrain». En rencontrant Doc, chef charismatique de la bande des Nortons, Whyte bénéficiait d'une certaine légitimité (en construction) lui permettant d'être accepté plus aisément par les autres membres de la bande et lui facilitant, par conséquent, le recueil de données.

Adler et Adler (1987), qui ont consacré un ouvrage au rôle du chercheur dans sa recherche, estiment que celui-ci doit prendre en considération ses propres émotions et expériences comme des variables centrales du processus d'analyse, soumettre les résultats de la recherche aux interviewés et s'inscrire dans un des trois niveaux de participation :

- L'observation participante périphérique. Le chercheur participe suffisamment au phénomène étudié pour être considéré comme un membre à part entière, sans incarner un rôle important au sein de celuici;

- L'observation participante active. Grâce à son implication personnelle, le chercheur tente de jouer un rôle important au sein du phénomène étudié, lui permettant de participer aux activités du groupe;

- L'observation participante complète. Le chercheur devient un membre investi de par son appartenance préalable au phénomène étudié ou par une conversion progressive lors de son immersion. 
Si elle permet d'accéder aux données « les plus profondes » et les plus riches d'un phénomène, l'implication personnelle, et de fait affective, peut avoir comme inconvénient de limiter la prise de recul du chercheur vis-à-vis de son objet d'étude. Lapassade (1991) s'interroge d'ailleurs sur ce rapport entre implication et distanciation :

Comment faire à la fois la part de l'implication dans la vie d'un groupe et d'une institution et la part de recul nécessaire, dit-on, si l'on veut rester un chercheur? Comment éviter de devenir soimême un indigène? (Lapassade, 1991, p. 33).

L'implication personnelle et affective du chercheur étant une condition nécessaire à toute recherche interprétative, la neutralité vis-à-vis du phénomène devient dénuée de sens. Cependant, le chercheur doit être conscient et gérer les risques qu'engendre cet investissement affectif.

Mitchell (1993, cité par Thiétart, 1999, p. 250) expose le «paradoxe de l'intimité ». L'intimité développée par le chercheur avec les acteurs lui ouvre les portes d'informations jusqu'ici inaccessibles. En revanche, cette intimité peut engendrer une prise de position du chercheur vis-à-vis des interviewés qui le limitera dans sa prise de recul et sa vision critique. Ce paradoxe de l'intimité doit être réfléchi par le chercheur en fonction de deux dimensions: la connaissance du terrain et l'implication affective du chercheur. De ces deux dimensions découle une typologie du rôle du chercheur au sein de son étude (Figure 1).

Bien que chaque profil de chercheur contienne des avantages et des contraintes, il apparaît, comme le souligne Mitchell (1993), que la position de l'allié avisé et compatissant constitue le rôle le plus riche. Cette position de l'allié avisé et compatissant a été l'objectif de cette démarche de recherche, concrétisé par une connaissance et un investissement croissant à partir de lectures, d'écoutes et d'échanges.

Au-delà de ce paradoxe de l'intimité, le chercheur fait également face à trois phénomènes de « contamination » des sources primaires (Mitchell, 1993) :

- Une contamination intragroupe. La participation régulière du chercheur devient un sujet de discussion entre les participants. L'introduction du chercheur par le parrain peut être perçue comme l'arrivée d'un « espion» au service du parrain. Les discours des participants pourront alors être « contaminés » par cette suspicion; 


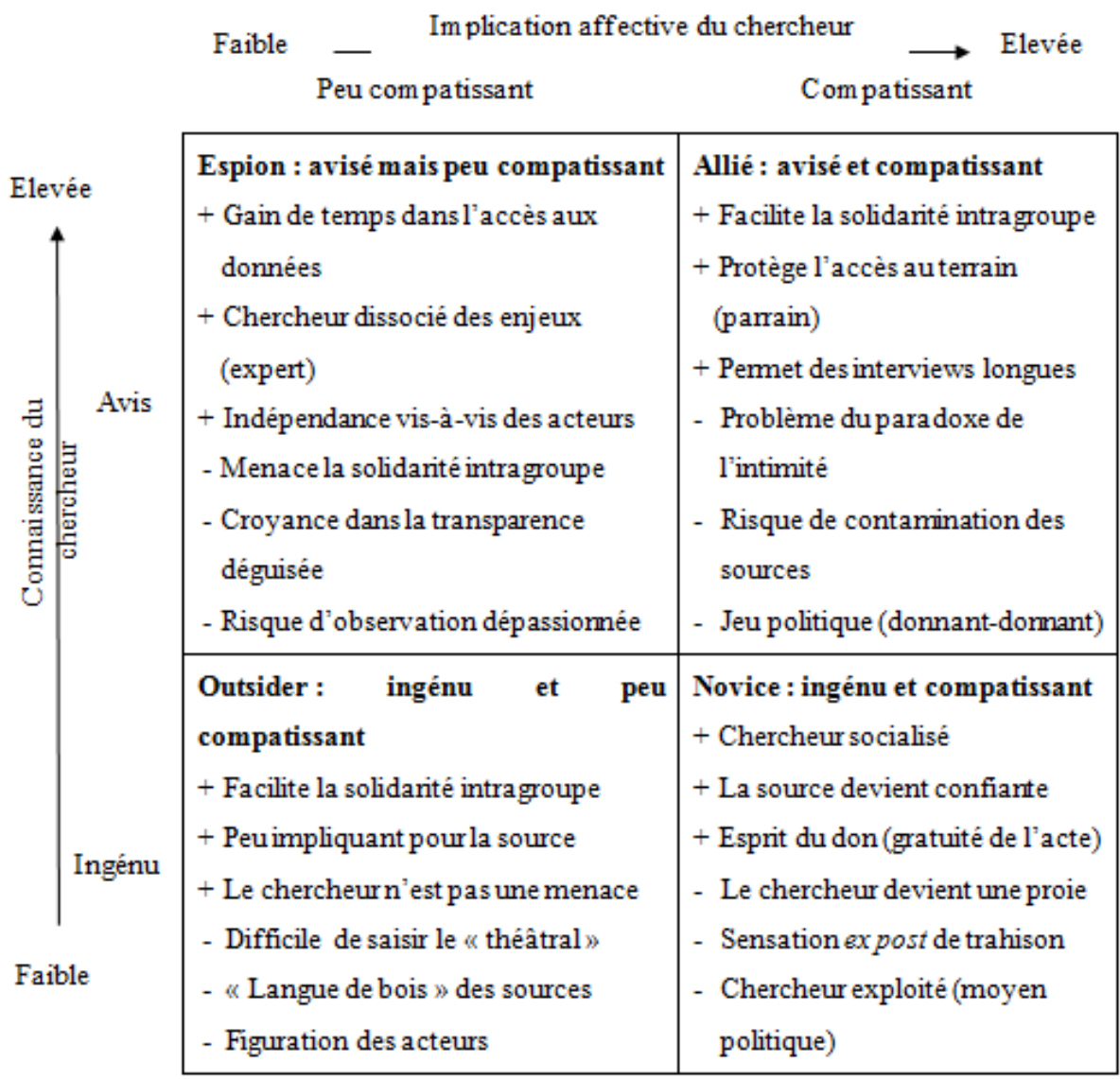

Figure 1. Perception du chercheur en fonction de son implication affective et de sa connaissance du terrain, inspirée de Mitchell (1993, cité par Thiétart, 1999, p. 250).

- Une contamination entre le chercheur et la population interviewée. Dans le cas où l'introduction du chercheur dans le groupe étudié s'est effectuée par l'intermédiaire d'un parrain, ce dernier peut jouir d'une influence, consciente ou inconsciente, sur le processus de recherche qu'il estime légitime en raison de son statut d'intermédiaire;

- Une contamination entre les sources primaires et les sources secondaires. Les participants peuvent influencer les données mises à disposition du 
chercheur en ne lui communiquant qu'une partie des données disponibles afin de « modeler » une situation qui peut être différente dans la réalité.

Bien que Mitchell (1993) mette en avant des facteurs de contamination, il ne propose pas de pistes afin d'en réduire les effets. Pourtant, ces questionnements liés à la position du chercheur sont fréquents, voire indispensables, animent constamment le travail du chercheur sur le terrain et influencent ce qui en ressort. La pluralité des outils de collecte et des sources de données, la tenue d'un journal de bord ainsi qu' une analyse interchercheurs, permettent d'atténuer ces phénomènes de contamination et de réduire l'impact de l'immersion émotionnelle du chercheur-ethnographe sur les résultats de sa recherche.

Ainsi, l'ethnographie apparaît comme un outil riche pour saisir la complexité des comportements contemporains de consommation mais également des problématiques de gestion plus large. Afin de rendre compte de la pertinence de l'outil ethnographique, la deuxième partie de cet article se base sur la présentation d'une recherche marketing centrée sur la compréhension de la consommation culturelle des jeunes au sein du mouvement rap français.

\section{Application de l'ethnographie : compréhension de la consommation culturelle au sein du mouvement rap en France}

La description de l'ethnographie qui compose cette deuxième partie est issue d'un travail doctoral soutenu en décembre 2011. S'inscrivant dans le champ de la Consumer Culture Theory (Arnould \& Thompson, 2005), et plus précisément dans une contextualisation du contexte (Askegaard \& TrolleLinnet, 2011), l'objectif initial était de comprendre les dynamiques consommatoires culturelles des jeunes au sein de mouvements culturels contemporains. Pour ce faire, cette recherche s'est proposée d'appréhender les pratiques de consommation à partir d'une lecture à un triple niveau : le jeune consommateur, le mouvement rap et l'environnement socioculturel. Cette recherche s'est alors articulée autour de différentes questions : quelles sont les pratiques et représentations des jeunes au sein de ces mouvements? Comment s'organisent ces pratiques? Comment se forment-elles? Quelles relations d'influence existe-t-il entre le jeune et l'environnement proche (mouvement culturel) et global (socioculturel)? Comment ces pratiques évoluent-elles? Afin d'apporter des éléments de réponse à ces questionnements, le choix s'est porté sur une approche ethnographique du mouvement rap en France qui s'est échelonnée sur une période de deux ans (Encadré 4). 
Originaire des quartiers défavorisés de New York, le rap, courant musical appartenant à la culture hip-hop, arrive en France au début des années 1980 par l'intermédiaire de voyageurs ayant rapporté de la musique du Bronx, puis par la voie de radios associatives et indépendantes. Après une première période durant laquelle les amateurs et les artistes rap représentent une communauté restreinte et peu connue, le rap, et plus largement le hip-hop, devient rapidement le courant culturel et artistique des quartiers de banlieues parisiennes, avant de gagner les grandes villes de province et de s'inscrire durablement dans le panorama culturel français. À son ascension médiatique et populaire s'ajoute alors une dimension marchande, alimentée par l'intérêt croissant des maisons de disques et d'entreprises culturelles, propulsant le rap en tête des ventes au début des années 1990.

Encadré 4. Le rap en France comme terrain ethnographique : bref rappel historique.

L'ethnographie du mouvement rap en France s'est faite selon les principes édictés par Arnould et Wallendorf (1994), à savoir l'immersion longue et personnelle du chercheur et la pluralité des méthodes de recueil de données (Annexe 1). Afin d'atténuer les facteurs de contamination édictés par Mitchell (1993), cette approche ethnographique s'est composée de plusieurs outils de collecte, de sources diverses de données et d'un journal de bord tenu quotidiennement.

Il convient maintenant de présenter, d'une part, les méthodes de collecte mobilisées ainsi que la démarche analytique retenue, puis, d'autre part, d'exposer les résultats ressortis de cette étude ethnographique.

Saisir la complexité du mouvement rap : emploi d'outils pluriels de collecte de données

Le choix d'une approche ethnographique a eu pour conséquence la mobilisation de plusieurs méthodes de collecte de données afin de «plonger » dans l'univers du rap. Au-delà des outils classiques présentés précédemment, l'avènement de la prédominance du média Internet nous a amené à employer la méthode netnographique. L'intégralité des outils de collecte de données et de la période de collecte est présentée en annexe (Annexes 2 et 3 ).

Observer pour mieux comprendre : lecture du rap en France

L'observation favorise l'interaction avec les répondants, la compréhension du terrain dans son milieu naturel ainsi que la collecte de données riches et diversifiées (écrites, sonores, visuelles, etc.). Bien que notre étude porte essentiellement sur le rap français, celui-ci reste fortement lié à la mouvance 
hip-hop dont il incarne une des disciplines artistiques ${ }^{4}$. Si la majeure partie des observations concerne directement le rap, des phases d'observation ont été sélectionnées dans un spectre plus large afin de préserver le contexte culturel dans lequel il a émergé.

Ainsi, l'appréhension du mouvement rap, des pratiques qui les composent, ainsi que de la structure et de l'évolution qui le caractérisent, s'est effectuée à partir de trois techniques d'observation :

- L'observation participante. L'étude du mouvement s'est réalisée à partir de séquences d'observation participante espacées dans le temps dans lesquelles le chercheur était, pour un temps, acteur direct lors d'émissions de radio ou de conférences portant sur le rap ou le hip-hop. Ces phases ont permis de confronter l'interprétation naissante du chercheur avec des membres du mouvement rap. Le chercheur, ici, est alors dans une observation participante périphérique (Adler \& Adler, 1987).

- L'observation non participante. Ces phases se sont déroulées lors d'évènements divers tels que des concerts de rap, des expositions liées au graffiti ou à la culture urbaine, des conférences en lien avec l'univers musical ou rapologique ainsi qu'à des festivals.

- L'observation mécanique. Outil complémentaire aux deux types d'observation précédents, l'observation mécanique consiste à recueillir des données visuelles, écrites ou sonores permettant d'enrichir l'analyse. À cette fin, l'ethnographie du mouvement rap s'est effectuée à travers la collecte de données et d'objets divers (vidéos, encarts [flyers], chansons, cadeaux Internet [goodies]) en vue d'affiner la compréhension du phénomène. On retrouve alors la volonté de Malinowski (1963) de penser l'ethnographie comme le récit minutieux de la culture étudiée.

\section{L'entretien comme moyen de saisir la perspective émique}

La compréhension du mouvement rap s'est aussi réalisée en sollicitant différents acteurs et jeunes membres intervenant et jouant un rôle plus ou moins important dans la vie et le développement de ce mouvement. Leurs regards de membres investis ont fourni des informations précieuses dans la constitution d'une analyse globale du rap et des jeunes qui s'y rattachent. Les personnes interrogées constituaient des informateurs susceptibles de fournir des données multiples. Nous avons considéré ces personnes de deux façons :

- Comme membre du mouvement rap. L'objectif était ici de retracer l'histoire personnelle de l'acteur ou du jeune ainsi que les facteurs qui l'ont conduit à « entrer» dans ce mouvement culturel. 
- Comme expert du mouvement rap. La personne interrogée incarnait un membre avisé du mouvement et apportait un regard expert sur sa structuration et son évolution. L'analyse était réalisée de concert avec les procédures de collecte. Les premiers résultats ressortis étaient alors régulièrement soumis à l'avis de ces interlocuteurs privilégiés.

Les entretiens ont ainsi été réalisés auprès de professionnels du secteur musical, d'acteurs médiatiques de la scène rap, d'artistes et de jeunes amateurs de rap.

Les prises de contact avec les personnes interrogées se sont effectuées à partir de rencontres lors des séquences d'observation, de conseils donnés par des interlocuteurs et par mail.

Netnographie : appréhension de la partie numérique et virtuelle du rap

Bien que le rap soit apparu alors qu'Internet n'était qu'à ses balbutiements, force est de constater que le rap français, et les membres qui en font partie, se sont appropriés ce nouveau média pour communiquer, diffuser et échanger. L'approche netnographique a donc eu pour objectif de saisir cette dimension virtuelle du mouvement.

Pour ce faire, une étude prolongée de différents sites Internet a été entreprise. La recherche par mots clés sur des moteurs de recherche, ainsi que des informations recueillies à l'aide d'autres outils, ont facilité la reconnaissance des sites et forums de discussion importants de la scène rap. Leur sélection s'est effectuée selon les critères édictés par la littérature (Kozinets, 2002). Quatre sites ont été intégrés de façon durable dans l'approche netnographique, mais d'autres sites Internet ont été consultés à plusieurs reprises sans avoir fait l'objet d'une analyse continue. Outre le suivi régulier de ces places virtuelles, l'approche netnographique a également été entreprise à partir d'informations recueillies et de discussions menées via le réseau social Facebook.

L'immersion personnelle du chercheur s'est traduite par la pratique régulière, voire quotidienne, d'activités en lien avec le mouvement rap comme l'écoute régulière de musique (plateforme numérique, achats de $\mathrm{CD}$, etc.), la lecture d'ouvrages et de magazines dédiés au rap ou à la mouvance hip-hop, et la participation à des concerts et à des festivals. À mi-chemin entre une perspective introspective émotionnelle et une nécessaire dimension réflexive, cette immersion s'est traduite par la prise de notes constantes de sentiments, remarques, idées et émotions dans un journal de bord. 
Ces divers outils de recueil de données ont permis la constitution d'un corpus de données riche et diversifié qui a fait l'objet d'un double processus analytique :

- Une analyse de contenu thématique a été effectuée de façon manuelle et à l'aide du logiciel NVivo afin de mettre en lumière des catégories et des métacatégories d'une part, et de comprendre les pratiques, la structuration du mouvement et les interactions entre les différents niveaux d'observation (entre le jeune, le mouvement et l'environnement socioculturel) d'autre part;

- Une analyse chronologique afin de retracer l'histoire à la fois des jeunes membres, du mouvement rap et des relations d'influences entre eux. Cette analyse diachronique a permis la réalisation de récits retraçant, à un niveau microsocial, le parcours des jeunes interrogés et, à un niveau macrosocial, la place du mouvement rap dans l'environnement culturel et sociétal français.

Le dernier développement de cette partie consacrée à l'application de l'ethnographie à la compréhension des dynamiques consommatoires au sein du rap français s'articule autour des résultats ressortis de cette investigation.

De la consommation du jeune membre à la place du rap dans le paysage culturel : présentation des résultats de l'ethnographie du rap

L'ethnographie du mouvement rap en France et le récit descriptif qui en est le fruit ont mis à jour des thèmes permettant d'apporter des éléments de compréhension aux questionnements originels de cette recherche. Les résultats de cette recherche s'articulent autour des trois pôles suivants : la consommation du jeune membre, la structuration du mouvement rap et son évolution.

Consommation du jeune : le style comme dénominateur commun des membres

Au-delà de l'importance des pairs dans l'entrée et la participation progressive au mouvement, il ressort de cette ethnographie des dynamiques consommatoires communes, partagées par l'ensemble des membres, de façon plus ou moins intensive. Ces signes ostentatoires fédérateurs, regroupés autour d'un style, ont pour fonction de permettre au jeune de revendiquer son appartenance au mouvement ; ils peuvent être répartis en cinq formes :

- La musique. Elle incarne la toile de fond culturelle du mouvement, son porte-drapeau. La musique représente l'essence originelle du mouvement rap et la pratique permettant au jeune d'intégrer cet univers culturel;

- Le vêtement. La revendication de l'appartenance au mouvement rap passe également par un style vestimentaire, traduction concrète et 
tangible de l'adhésion du jeune à ce mouvement culturel particulier (ex : baggy, basket, casquettes, etc.);

- Les marques. Dans ce désir d'ostentation et de reconnaissance, les marques incarnent également un vecteur symbolique. Le jeune construit son identité de membre du mouvement rap à partir de marques de luxe (Lacoste, Dolce Gabbana, etc.), de marques de sport (Nike, Adidas, etc.), et de marques spécifiques créées par et pour les membres du mouvement rap (Wrung, Com8, FuBu', etc.);

- Le langage. L'affichage de son appartenance passe, en outre, par des pratiques langagières particulières, articulées autour d'un argot spécifique au mouvement rap (ex: le verlan), mais également d'expressions inspirées de l'environnement numérique;

- Les rituels. Enfin, les rituels participent également au sentiment commun des membres d'appartenir au mouvement rap. Il apparaît que ces rituels sont à la fois individuels (pratique quotidienne du jeune membre comme l'écoute de musiques, l'habillement) et collectifs (concerts, moments d'écoutes collectives).

Influences réciproques entre les jeunes membres et leur environnement

À une échelle plus large, on observe que les jeunes membres du mouvement rap entretiennent des relations d'influences réciproques avec l'environnement dans lequel ils négocient et partagent leurs comportements de consommation :

- À un niveau local. Au sein de contextes proches et de territoires locaux, les jeunes membres du mouvement nourrissent des interactions réciproques. La famille, les établissements scolaires (collège, lycée), les territoires (le quartier, la ville, le département, la région) sont le théâtre de pratiques communes où les jeunes sont à la fois récepteurs et créateurs de sens;

- À un niveau global. Au-delà de cette proximité géographique, les jeunes membres du mouvement rap nourrissent des influences mutuelles au sein d'univers plus larges. À titre d'illustration, les jeunes sont à la fois récepteurs d'informations médiatiques (radio, télévision) et créateurs de contenus (réseaux sociaux, téléchargement). De même, dans l'univers marchand, les jeunes sont à la fois des consommateurs actifs et des producteurs légitimes de produits culturels (création de marques spécifiques) susceptibles de dépasser l'univers du rap.

Ces influences réciproques, qu'elles soient locales ou globales, ont contribué à l'évolution du mouvement rap en France et aux comportements de consommation qui le composent. 


\section{De l'authenticité à la banalisation : évolution du mouvement rap en France}

Le dernier pan composant les résultats de cette ethnographie du rap est consacré à l'évolution du mouvement rap. Cette évolution semble se composer de trois phases principales :

- Naissance et détournement. L'apparition du mouvement rap en France et des comportements de consommation qui s'y rattachent ne sont pas le fruit d'une création ex nihilo, mais le résultat d'actes de détournements (artistiques, symboliques) d'éléments préexistants;

- Diffusion et dispersion. L'originalité du mouvement rap, de ses symboles et de ses produits, concourt à une diffusion du mouvement rap par l'intérêt médiatique et marchand qu'il suscite. On observe également une dispersion (géographique, sociale, sexuée et générationnelle) sortant les éléments structurant du mouvement rap de leur contexte originel (quartiers populaires de Paris et Marseille, masculin);

- Récupération et banalisation. Cette croissance du mouvement rap et des comportements de consommation liés à ce mouvement provoque une récupération commerciale et publicitaire ayant pour conséquence de banaliser le mouvement rap et d'en faire un univers culturel supplémentaire dans le paysage culturel français.

Les résultats de cette approche ethnographique, présentés ici à partir de trois principales catégories, ont été par la suite confrontés à la littérature en comportement du consommateur afin d'apporter des éléments nouveaux dans la compréhension de la consommation culturelle du jeune et de proposer des perspectives managériales pertinentes à des organisations susceptibles de s'intéresser aux jeunes ou à des univers culturels émergents.

Ces résultats, certes synthétiques, démontrent l'intérêt de l'ethnographie dans le cadre de réflexions portant sur les comportements de consommation à partir d'une prise en compte du consommateur, mais également du contexte dans lequel il agit, partage et s'inspire. Ils ont de plus permis de proposer des implications managériales à destination de différents acteurs, comme la nécessité, pour les artistes, de proposer, au-delà de la musique, un univers plus large, source de revenus supplémentaires. Cette étude émet également des remarques à destination des entreprises et des marques désireuses de répondre aux besoins des membres de ce type de mouvement culturel, comme la recherche de légitimité et l'adaptation du territoire de marque. Enfin, l'étude longitudinale offre une lecture dynamique et prédictive des mouvements culturels, permettant ainsi aux acteurs économiques et publics d'adapter leurs propositions en fonction des stades de développement du mouvement. 


\section{Conclusion}

À partir d'une exposition des dynamiques consommatoires du jeune, de la façon dont elles s'articulent et évoluent, cette ethnographie du rap et les résultats qui en ressortent ont fait ressortir des implications managériales à l'intention d'organisations (entreprises, associations) et de pouvoirs publics dont le jeune et les pratiques culturelles incarnent la cible. De ce fait, l'ethnographie apparaît comme une méthode d'investigation pertinente et riche dans le cadre de problématiques de gestion. Si cet article traite de l'approche ethnographique, de ses principes et de son intérêt dans le cadre d'une recherche en marketing en particulier, elle est aujourd'hui également une perspective méthodologique grandissante dans les sciences de gestion. En effet, un nombre croissant de travaux traite de problématiques liées aux ressources humaines (Raulet-Croset, 2003), ou plus largement de 1'entreprise (Lambelet, 2003) ${ }^{6}$, à partir d'une investigation ethnographique. Ainsi, sa mise en place dans le cadre de problématiques organisationnelles ou consommatoires permettra, outre la mise en lumière de nouvelles pratiques ou de représentations, de consolider les approches qualitatives en général, et la démarche ethnographique en particulier.

\section{Notes}

1 À partir d'études empiriques visant à comprendre les dimensions ethniques et collectives de communautés issues de l'immigration de Chicago puis des grandes villes des États-Unis, le courant de recherche attaché à l'École de Chicago adaptera la démarche ethnographique à un contexte social et culturel contemporain des communautés américaines.

${ }^{2}$ Selon Kozinets (2002), la mise en place d'une netnographie suppose le recours à plusieurs critères de sélection afin de choisir les sites Internet les plus pertinents (proximité avec la question de recherche, nombre de membres inscrits sur les forums et de messages publiés). À cela, nous avons ajouté la recommandation de sites par des interlocuteurs privilégiés.

${ }^{3}$ Il convient de préciser ici que le terme indigène, s'il est utilisé par Malinowski afin de qualifier la population étudiée, ne l'est pas dans cette démarche de recherche. Nous l'entendons ainsi comme un qualificatif permettant de distinguer le chercheur de la population considérée.

${ }^{4}$ Né dans les quartiers défavorisés de New York, le hip-hop représente une mouvance artistique plurielle, composée de différentes disciplines telles que le breakdance (la danse), le graffiti, le deejaying et le rap.

${ }^{5} \mathrm{La}$ marque $\mathrm{FuBu}$ est, à ce titre, un bon exemple pour illustrer la création de marques spécifiques au sein de la culture hip-hop puisqu'elle signifie « For Us, By Us». 
${ }^{6}$ À ce titre, le Journal des Anthropologues publie, dès 1991, un dossier spécial intitulé «Ethnologie de l'entreprise» afin de proposer une vision théorique et empirique de l'outil ethnographique.

\section{Références}

Abélès, M. (2008). Anthropologie de la globalisation. Paris : Payot.

Adler, P. A., \& Adler, P. (1987). Membership roles in field research. Newbury Park : Sage.

Appaduraï, A. (1990). Disjuncture and difference in the global economy. Dans M. Featherstone (Éd.). Global culture: nationalism, globalization and modernity (pp. 295-310). Londres : Sage.

Arnould, E., \& Thompson, C. J. (2005). Consumer culture theory (CCT) : twenty years of research. Journal of Consumer Research, 31(4), 868-882.

Arnould, E., \& Wallendorf, M. (1994). Market-oriented ethnography : interpretation building and marketing strategy formulation. Journal of Marketing Research, XXXI, 484-504.

Askegaard, S., \& Trolle-Linnet, J. (2011). Vers une épistémologie de la théorie de la culture du consommateur (CCT) : phénoménologie, structure et contexte du contexte. Perspectives culturelles de la consommation, 1, 1-52.

Badot, O. (2005). Esquisse des fonctions socio-anthropologiques du commerce et de la distribution : les cas McDonald's, West Edmonton Mall et Wall Mart (Thèse de doctorat inédite). Faculté des Sciences Humaines et Sociales de La Sorbonne, Paris, France.

Badot, O., Carrier, C., Cova, B., Desjeux, D., \& Filser, M. (2009). L'ethnomarketing : un élargissement de la recherche en comportement du consommateur à l'ethnologie. Recherche et applications en marketing, 24(1), 93-111.

Belk, R. W. (1987). The role of the odyssey in consumer behavior and in consumer research. Advances in Consumer Research, 14, 357-361.

Belk, R. W., Wallendorf, M., \& Sherry, F. Jr. (1989). The sacred and the profane in consumer behavior: theodicy on the odyssey. Journal of Consumer Research, 16, 1-38.

Blackman, S. (2005). Youth subcultural theory: a critical engagement with the concept, its origins and politics, from the Chicago School to postmodernism. Journal of Youth Studies, 8(1), 1-20. 
Cohen, P. (1972). Subcultural conflict and working class community [Document de travail dans les études culturelles]. Birmingham : Centre for Contemporary Cultural Studies (CCCS).

Copans, J. (1996). Introduction à l'ethnologie et à l'anthropologie. Paris : Nathan Université.

Coulon, A. (1992). L'école de Chicago. Paris: Presses universitaires de France.

Dion, D. (2008). À la recherche du consommateur : nouvelles techniques pour mieux comprendre le client. Paris : Dunod.

Ezan, P. (2009). De l'intérêt de la démarche ethnographique pour comprendre les pratiques de consommation des enfants. Recherche et applications en marketing, 21(4), 77-95.

Geertz, C. (1973). Interpretation of cultures. New York : Basic Books.

Geertz, C. (1998). La description dense. Vers une théorie interprétative de la culture. Enquête, 6. Repéré à http://enquete.revues.org/1443.

Hall, S., \& Jefferson, T. (1976). Resistance through rituals : youth subcultures in post-war Britain. Londres : Routledge.

Hannerz, U. (2003). Being there... and there... and there! Reflections on multisite ethnography. Ethnography, 4(2), 201-216.

Hebdige, D. (1979). Sous-culture. Le sens du style. Paris: Éditions La Découverte.

Journal des Anthropologues. (1991, Mai). Dossier "Ethnologie de l'entreprise". Association française des anthropologues, 43-44.

Kates, S. M. (2002). The protean quality of subcultural consumption : an ethnographic account of gays consumers. Journal of Consumer Research, 29(3), 383-399.

Kjelgaard, D., \& Askegaard, S. (2006). The glocalization of youth culture : the global youth segment as structures of common difference. Journal of Consumer Research, 33, 231-247.

Kjeldgaard, D., Faurholt Csaba, F., \& Ger, G. (2006). Grasping the global : multi-sited ethnographic market studies. Dans R. K. Belk (Éd.), Handbook of qualitative research methods in marketing (pp. 521-553). Cheltenham: Edward Elgar Publishing.

Kozinets, R. V. (1997). I want to believe : a netnography of the X-Files' subculture of consumption. Advances in Consumer Research, 24, 470-475. 
Kozinets, R. V. (2002). The field behind the screen : using netnography for marketing research in online communities. Journal of Marketing Research, 39, 61-72.

Lambelet, A. (2003). Un ethnologue en entreprise: entre séduction et révélation. Ethnographie.org, 3 . Repéré à http;//www.ethnographiques.org/2003/Lambelet/html

Lapassade, G. (1991). L'ethnosociologie, les sources anglo-saxonnes. Paris : Méridiens Klyncksieck.

Laplantine, F. (2006). La description ethnographique. Paris : Armand Colin.

Malinowski, B. (1963). Les Argonautes du Pacifique occidental. Paris: Gallimard.

Malinowski, B. (1985). Journal d'ethnographe. Paris : Le Seuil.

Marcus, G. E. (1995). Ethnography in/of the world system : the emergence of multi-sited ethnography. Annual Review of Anthropology, 24, 95-117.

Mariampolsky, H. (2006). Ethnography for marketers : a guide to consumer immersion. London: Sage.

Mauss, M. (1926). Manuel d'ethnographie. Paris : Éditions Sociales.

Mitchell, R. G. (1993). Secrecy and fieldwork. Newbury Park : Sage.

Peñaloza, L. (1994). Atravesando fronteras/border crossings: a critical ethnographic study of the consumer acculturation of mexican immigrants. Journal of Consumer Research, 21, 32-53.

Peñaloza, L. (1999). Just doing it. A visual ethnography of spectacular consumption behavior at Nike Town. Consumption, Markets and Culture, 2(4), 337-400.

Raulet-Croset, N. (2003). La méthode ethnographique appliquée aux ressources humaines. Dans J. Allouche (Éd.), Encyclopédie des ressources humaines (pp. 878-887). Paris : Vuibert.

Ritson, M., \& Elliot, R. (1999). The social uses of advertising : an ethnographic study of adolescent advertising audiences. Journal of Consumer Research, 26, 260-277.

Schouten, J. W., \& McAlexander, J. H. (1995). Subcultures of consumption : an ethnography of the new bikers. Journal of Consumer Research, 22, 43-61.

Thiétart, R. A. (Éd.). (1999). Méthodes de recherche en management. Paris : Dunod. 
72 ReCHERCHES QuALITATIVES / Vol. 32(2)

Van Maanen, J. (1979). The fact of fiction in organizational ethnography. Administrative Science Quartely, 24(4), 539-550.

Whyte, W. F. (1955). Street corner society: the social structure of an italian slum. Chicago : The University of Chicago Press.

Baptiste Cléret est Maître de conférences à l'Université de Rouen et a obtenu son doctorat en Sciences de Gestion, mention Marketing, en décembre 2011. Ses recherches s'inscrivent dans le champ de la Consumer Culture Theory et portent principalement sur les dynamiques consommatoires des jeunes, les pratiques artistiques et culturelles et les méthodes qualitatives. 
Annexe 1

Applications des conditions d'étude ethnographique proposées par Arnould et Wallendorf (1994) au cas du mouvement rap

\begin{tabular}{|c|c|}
\hline $\begin{array}{c}\text { Caractéristiques d'une ethnographie } \\
\text { (Arnould \& Wallendorf, 1994, } \\
\text { p. 485) }\end{array}$ & $\begin{array}{c}\text { Application à l'approche } \\
\text { ethnographique du mouvement rap en } \\
\text { France }\end{array}$ \\
\hline $\begin{array}{l}\text { "L'ethnographie donne la priorité à la } \\
\text { collecte systématique de données et à } \\
\text { l'enregistrement d'actions humaines } \\
\text { dans leurs cadres naturels. » }\end{array}$ & $\begin{array}{l}\text { Observations réalisées lors d'événements } \\
\text { liés au mouvement rap (conférences, } \\
\text { concerts, festivals, émissions de radio } \\
\text { etc.). }\end{array}$ \\
\hline $\begin{array}{l}\text { «La recherche ethnographique } \\
\text { implique une participation } \\
\text { expérientielle étendue de la part du } \\
\text { chercheur, dans un contexte culturel } \\
\text { spécifique. » }\end{array}$ & $\begin{array}{l}\text { L'implication personnelle du chercheur } \\
\text { s'est traduite par une démarche } \\
\text { introspective dans laquelle nous avons } \\
\text { pris part à différentes activités liées au } \\
\text { mouvement rap (écoute de musique et de } \\
\text { radio, lecture d'ouvrages et de revues, } \\
\text { concerts, visionnage de documentaires } \\
\text { etc.). }\end{array}$ \\
\hline $\begin{array}{l}\text { "L'ethnographie produit des } \\
\text { interprétations des comportements que } \\
\text { les personnes étudiées et l'audience } \\
\text { trouvent crédibles. » }\end{array}$ & $\begin{array}{l}\text { Le contact régulier avec certains } \\
\text { membres du mouvement rap a permis de } \\
\text { soumettre fréquemment les avancées } \\
\text { descriptives et interprétatives de la } \\
\text { recherche à leurs connaissances du rap, } \\
\text { orientant ainsi les phases de collecte } \\
\text { ultérieures et la description du } \\
\text { mouvement. De plus, une fois retranscrit, } \\
\text { chaque entretien était envoyé aux } \\
\text { personnes concernées. }\end{array}$ \\
\hline $\begin{array}{l}\text { "L'ethnographie implique } \\
\text { l'incorporation de sources multiples de } \\
\text { données et une stratégie de recherche } \\
\text { recommandée dans d'autres traditions } \\
\text { de sciences sociales. » }\end{array}$ & $\begin{array}{l}\text { Méthodes de collecte multiples : étude } \\
\text { netnographique, observations } \\
\text { participantes et non participantes, } \\
\text { entretiens ethnographiques, introspection. }\end{array}$ \\
\hline
\end{tabular}


74 ReChERChes QuALit ATIVES / Vol. 32(2)

Annexe 2

Application des outils de collecte de données sélectionnés

\begin{tabular}{|c|c|}
\hline Outils de collecte sélectionnés & Applications \\
\hline Observations & $\begin{array}{c}22 \text { phases d'observations non } \\
\text { participantes (concerts, conférences, } \\
\text { festivals, magasins culturels, lieux } \\
\text { publics); } \\
3 \text { phases d'observations participantes } \\
\text { (conférence, émissions de radio). }\end{array}$ \\
\hline Entretiens & $\begin{array}{l}8 \text { entretiens réalisés avec des jeunes } \\
\text { membres, professionnels et artistes du } \\
\text { mouvement rap. }\end{array}$ \\
\hline Netnographie & $\begin{array}{l}4 \text { sites Internet sélectionnés } \\
\text { (222 messages étudiés et incorporés } \\
\text { au sein du corpus de données). }\end{array}$ \\
\hline Immersion personnelle du chercheur & $\begin{array}{c}\text { Écoute de musique, lecture } \\
\text { d'ouvrages et de magazines dédiés au } \\
\text { rap, visionnage de films et de } \\
\text { documentaires, participation à des } \\
\text { évènements dédiés (concerts, } \\
\text { festivals). }\end{array}$ \\
\hline
\end{tabular}


Annexe 3

Chronologie de l'ethnographie du mouvement rap en France

\begin{tabular}{|c|c|}
\hline $\begin{array}{c}\text { Septembre } \\
2009\end{array}$ & 30/09 : Inscription sur le site Internet Booska-p \\
\hline $\begin{array}{l}\text { Octobre } \\
2009\end{array}$ & $\begin{array}{l}\text { 06/10 : Inscription sur le site Internet de Skyrock } \\
\text { 13/10 : Exposition Né dans la rue, Fondation Cartier, Paris } \\
\text { 20/10 : Concert Oxmo Puccino, Hangar 23, Rouen } \\
\text { 26/10 : Conférence de Christian Béthune sur les racines du } \\
\text { Hip-hop, Canteleu }\end{array}$ \\
\hline $\begin{array}{l}\text { Novembre } \\
2009\end{array}$ & $\begin{array}{l}\text { 05/11 : Soirée cinéma hip-hop, cinéma UGC, Rouen } \\
\text { 17/11 : Inscription au webzine } A b c d r d u \text { son }\end{array}$ \\
\hline $\begin{array}{l}\text { Décembre } \\
2009\end{array}$ & 07/12 : Entretien avec E., producteur indépendant, Rouen \\
\hline $\begin{array}{c}\text { Janvier } \\
2010\end{array}$ & $\begin{array}{l}\text { 06/01 : Observation dans } 3 \text { magasins rap à Chatelet les Halles, } \\
\text { Paris } \\
22 / 01 \text { : Entretien avec V., rappeur, Rouen }\end{array}$ \\
\hline $\begin{array}{l}\text { Février } \\
2010\end{array}$ & $\begin{array}{l}\text { 10/02 : Rencontre Lydie Milhet, responsable Communication } \\
\text { de la Fnac, Rouen } \\
\text { 16/02 : Conférence Les publics du rap, Festival Hip } \\
\quad \text { Opsession, Nantes } \\
\text { 17/02 : Exposition Korsé (graffiti), Festival Hip Opsession, } \\
\text { Nantes } \\
\text { 17/02: Observation de lieux à graffiti, Nantes }\end{array}$ \\
\hline Mars 2010 & $\begin{array}{l}\text { 01/03 : Observation, répétition Vîrus et Dj Bachir, Louviers } \\
\text { 09/03 : Concert de Vîrus, Lundis du Kalif, Rouen }\end{array}$ \\
\hline Avril 2010 & $\begin{array}{l}\text { 21/04 : Observation, Hip Hop Afternoon, Festival des cultures } \\
\text { urbaines, Canteleu }\end{array}$ \\
\hline Mai 2010 & 04/05 : Soirée concerts West Coast, Shari Vari, Rouen \\
\hline
\end{tabular}


Annexe 3

Chronologie de l'ethnographie du mouvement rap en France (suite)

\begin{tabular}{|c|c|}
\hline Juin 2010 & $\begin{array}{l}\text { 24/06 : Concert MCS et Sly Johnson, Paris Hip-hop Festival, } \\
\text { Ivry sur Seine } \\
\text { 28/06 : Entretien avec V., rappeur, Rouen } \\
\text { 29/06 : Conférence Kevin Liles, Paris Hip-hop Festival, Paris }\end{array}$ \\
\hline Juillet 2010 & $\begin{array}{l}\text { 01/07 : Participation, Table ronde La diffusion du rap en } \\
\text { France, Paris Hip-hop Festival, Paris } \\
\text { 20/07 : Réunion avec Vîrus sur le développement de son } \\
\text { projet associatif }\end{array}$ \\
\hline Août 2010 & $\begin{array}{l}\text { 09/08 : Repas avec Bachir et Vîrus, Rouen } \\
\text { 19/08: Observation sur les coins à graffitis de Rouen } \\
\text { 20/08 : Entretien avec T, manager de MCS } \\
\text { 27/08 : Concert Cypress Hill, Festival Rock en Seine, Paris }\end{array}$ \\
\hline $\begin{array}{l}\text { Octobre } \\
2010\end{array}$ & $\begin{array}{l}\text { 23/10 : Participation émission Edutainment, Radio HDR, } \\
\text { Rouen }\end{array}$ \\
\hline $\begin{array}{c}\text { Novembre } \\
2010\end{array}$ & $\begin{array}{l}\text { 05/11 : Concert Showcase MCS à la Fnac, Rouen } \\
\text { 05/11 : Concert MCS + Hocus Pocus, Notre-Dame-de- } \\
\text { Gravenchon } \\
\text { 10/11 : Concert de Wax Taylor + orchestre symphonique, } \\
\text { Théâtre des Arts, Rouen }\end{array}$ \\
\hline $\begin{array}{c}\text { Décembre } \\
2010 \\
\end{array}$ & 17/12 : Concert MCS, Café de la Danse, Paris \\
\hline $\begin{array}{c}\text { Janvier } \\
2011\end{array}$ & $\begin{array}{l}\text { 08/01 : Participation émission Edutainment, Radio HDR, } \\
\text { Rouen } \\
\text { 11/01 : Entretien G. C., Rédacteur en chef de Rap Mag, Paris } \\
\text { 25/01 : Entretien F. M., Animateur Skyrock, Paris } \\
\text { 26/01 : Exposition Jean-Michel Basquiat, Musée d'Arts } \\
\quad \text { Modernes, Paris } \\
\text { 31/01 : Entretien M., gérant du site Les Puces de Clicli, Paris }\end{array}$ \\
\hline
\end{tabular}


Annexe 3

Chronologie de l'ethnographie du mouvement rap en France (suite)

\begin{tabular}{|c|c|}
\hline $\begin{array}{l}\text { Février } \\
2011\end{array}$ & $\begin{array}{l}\text { 05/02 : Observation sur les Puces de Clignancourt } \\
\text { 18/02 : Concert de Youssoupha, Festival Hip-hop en Seine, } \\
\text { Rouen }\end{array}$ \\
\hline Avril 2011 & $\begin{array}{l}\text { 08/04 : Concert de Pigeon John, salle 106, Rouen } \\
\text { 19/04 : Soirée concerts Expérience rap, salle 106, Rouen } \\
\text { 20/04 : Concert Akhenaton et Faf Larage, salle 106, Rouen }\end{array}$ \\
\hline Mai 2011 & $\begin{array}{l}\text { 06/05 : Exposition Poska Nostra, Saint-Etienne du Rouvray } \\
\text { (76) }\end{array}$ \\
\hline Juillet 2011 & 22/07 : Entretien F., amateur de rap, Rouen (76) \\
\hline $\begin{array}{l}\text { Septembre } \\
2011\end{array}$ & 16/09 : Entretien M. amateur de rap, Rouen (76) \\
\hline
\end{tabular}

